\title{
Study of Classification of Human Emotion Features from EEG Signals at Minimum Free Energy Operated Effortlessly
}

\author{
Hong Yu, Sean-Paul Tomer, Marcus Perla, and Yvette Yu
}

\begin{abstract}
With the rapid development of various EEGbased features, PC machine robots can apply their fast massive parallel electronic computing AI capability to BCI systems. However, the machines do not have the human-equivalent chemical macromolecular hormone signals to understand human emotions. Machines cannot test people who are home alone with their loneliness, depression, drowsiness, happiness or remark the real hints through human being communication. But a minimum free energy of EEG discrete wavelet transform can indicate human emotion features and human being trend action. In this paper, we focus on EEG discrete wavelet transform at minimum free energy compared to operating effortlessly in visualization algorithm. In addition, we discuss EEG-based brain activities with the discrete wavelet energy spectrum for clinic treatment.
\end{abstract}

Key words - EEG, human-equivalent, hormone, features, emotion, minimum free energy, machines.

\section{INTRODUCTION}

In recent years, more researchers focused on brain computer interface (BCI) systems, especially on electroencephalogram (EEG) based emotion recognition. The various approaches and real-world applications of BCI systems have been enveloped with the rapid development of dry electrode, digital signal processing and computermachines even human emotions can be recognized through words, voice, gesture, facial expression and body language. Therefore, most of the effort has been dedicated to the design of BCI systems by means of innovative interfaces such as voice, vision and other input devices in virtual reality. As one of the approaches for various brainwaves, the electroencephalogram (EEG) can represent the brain activities and brainwaves traces as well. In general, the noninvasion and invasion methodology have been applied to EEG data acquisition. The non-invasive brain-imaging methodology records the brain's activities at the surface of the scalp. Another method is invasive brain-imaging that records the brainwave by the dry electrodes virtually [1].

Since the human brain is estimated to contain 100 billion neurons, the substantial amount of brain neuron activities need to be tracked by PC machines in their fast massive parallel electronic computing artificial intelligence (AI) capability. According to the brain activities and EEG signal frequency correspondent ranges, brainwaves can be classified to delta waves $(<4 \mathrm{~Hz})$, theta waves $(4-7 \mathrm{~Hz})$, alpha waves $(8-$ $12 \mathrm{~Hz})$, beta waves $(13-30 \mathrm{~Hz})$ and gamma waves $(>31 \mathrm{~Hz})$ in frequency ranges. These brain signals usually associate

\footnotetext{
Submitted on September 23, 2021.

Published on October 14, 2021.

Dr. Hong Yu, IEEE senior member, Engineering Technology Department,

Fitchburg State University, USA

(e-mail:yhong2006@gmail.com).
}

with: (1) deep dreamless sleep or loss of body awareness in delta waves (2) deep mediation or relaxation in theta waves (3) calm relaxed yet alert state in alpha waves (4) activities, busy thinking, active concentration, arousal and cognition in beta waves (5) higher mental activity including perception, problem solving and conscious in gamma waves. Fig. 1 illustrates the brain waveform frequency ranges and the brain activities.

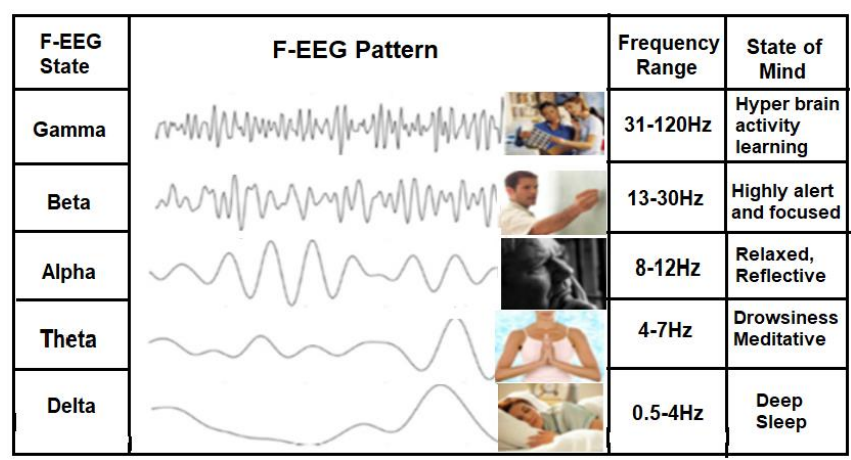

Fig. 1. Brain waveform frequency ranges and the actives.

For EEG data acquisition, the non-invasive EEG detection methodology is based on the recordings of brain wavelet from the surface of human forehead as the processor for the functional-EEG data. The main disadvantage of non-invasion to EEGs recording is that it does not have highly spatial resolution. The wavelets have been limited since the spatial and temporal resolution of EEGs are limited due to the overlapping electrical activities generated by different cortical areas [2].

For another EEG detection methodology, very small electrodes are directly implanted into the human brain. This methodology has been defined as the invasive detection EEG methodology. The probe is about the size of a large coin and can be fully embedded in the skull which is how Neuralink's robotic technology accomplished this [3]. The advantage of the invasive methodology is that these electrodes can be specifically targeted at arbitrary cerebral areas although accuracy decreases with implantation depth. The real-time interaction between the subject and the computer is an essential part of BCI systems. But it has to insert the devices into the human brain. Therefore, the non-invasive methodology has been commonly used for EEG data acquisition.

The rest of this paper is organized as follows. Section II

Sean-Paul Tomer, IEEE Student member, Engineering Technology Department, Fitchburg State University, USA.

Marcus Perla, IEEE Student member, Engineering Technology Department, Fitchburg State University, USA.

Yvett $\mathrm{Yu}$, senior consultant in Protiviti Golobel company, USA. She is Global Mindset Consulting Fellow and WST Fellow. 
surveys the classification algorithm of human emotions, patterns and feature extraction. Section III reviews EEG wavelet transform and contemporary methodology. Section VI illustrates the research methodology with the data analysis of the experiments. Section V discusses the future work, and conclusions are given in Section VI.

\section{SURVEY OF THE ClASSIFICATION ALGORITHMS AND PATTERNS}

In order to control a BCI system, the researchers and scientists have created multiple different brain activity patterns or models according to the data as represented by feature vectors such as the P300-BIC [4]. The performance of the pattern recognition depends on both the feature vector and classification algorithm employed. These feature vectors are designed to serve locked-in the patterns, and the algorithms aim at automatically detecting or estimating the properties of the feature vectors. After the pattern has been identified by the algorithms, it will translate the brain activity into the commands for a computer.

In the recent years, a classifier for a given set of feature vectors always deals with the problems of the vector-reduced dimensionality and the bias-variance tradeoff. According to the EEG feature signals, the classifier algorithms are divided into five different categories: linear classifiers, neural networks, nonlinear Bayesian classifiers, nearest neighbor classifiers, and combinations of classifiers[5]. The appropriate classifier with good feature extraction method should reduce the dimension of the input data as much as possible while keeping all the information necessary for the classification. Dimension reduction can then be performed by spatial filtering. Typically, the common spatial pattern method (CSP) determines spatial filters that maximize the temporal variance of data recorded under one condition and minimizes the temporal variance of data recorded under a second condition.

\section{EEG DESCRET WAVELET AND THE CONTEMPORARY METHODOLOGY REVIEW}

As it is well known, the ability to communicate with other people is one of the main factors that make human life enjoyable, because speech, gesturing or writing allow us to communicate with each other. BCI research develops systems that allow disabled users to communicate with artificial limbs or circumstances through EEG discrete wavelets transforms. In the following subsections we review the EEG signal processing, feature classifications and patterns that are typically used in BCI systems. In addition, we give a brief introduction to the EEG application issues in the methodology or algorithm.

\section{A. EEG discrete Wavelets Signal Processing}

In our previous research, the human-equivalent chemical macromolecular hormone signals can be transformed to discrete wavelets. Fig. 2 (a) illustrates EEGs discrete wavelets with frequency and magnitude properties.

Due to Hebb observation "wired together, fired together". Thus, the neurons population density of firing varies like a wave. The basic human emotions of loneliness, sadness, joy, happiness and fear can adapt to the electroencephalogram (EEG) as discrete brain wavelets. The discrete brain wavelets are shown in Fig. 2(c). Oscillatory activity in the EEG is classified into the different frequency bands or rhythms. Meanwhile, human daily activities or behaviors such as deep sleep, meditation, wakefulness and learning can be mapped into EEG patterns as well. It has been shown in Fig. 1.

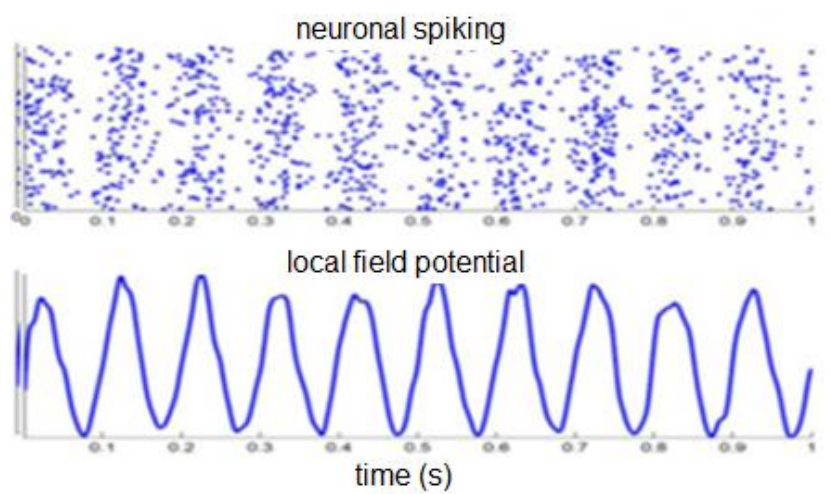

(a)

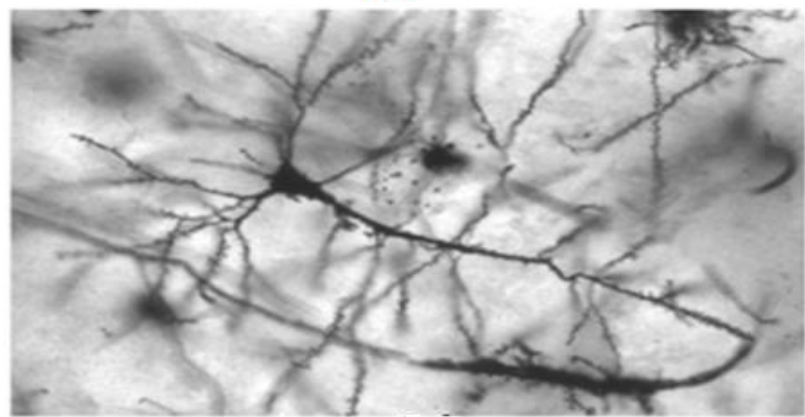

(b)

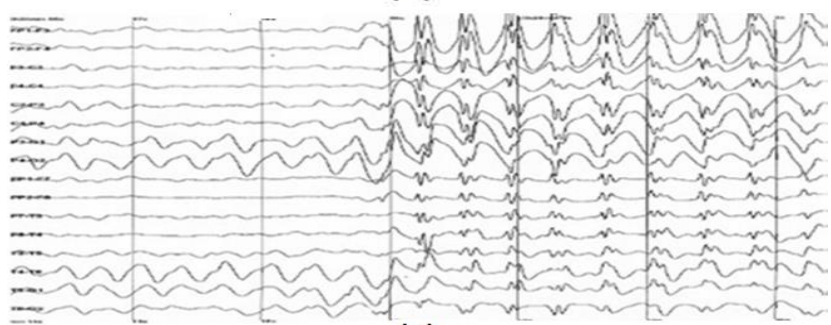

(c)

Fig. 2. (a) Neurons population fired in bundles, the population density of firing varies like a wave (b) Laser can burn off the axon feedback knot at dendrite (c) Time from the left to the right, the phase transition resulted in the epileptic seizure from excessive positive feedback gain instability of neuronal feedback.

The main aim to design a BCI system is to sense and evaluate the features of signals in a user's brain, which shows the intention of the user and sends the characters of signals to the external device that executes to fulfill the intention of the user. A BCI based system contains three sequential components: signal acquisition, signal processing, and control devices or feedback. Fig. 3 shows the flow block diagram of BCI system.

For EEG signal processing, it includes signal characteristic or feature extraction, classification and pattern recognition. The EEG signals are bandpass filtered with different bandwidth filters before submitting the signal data to the feature extraction and learning algorithms such as $0.1 \mathrm{~Hz} \sim$ $5 \mathrm{~Hz}$ bandwidth with a $4^{\text {th }}$ order butterworth filter. The higher order bandpass filter for EEG signal possesses the property to preserve both magnitude and phase information. 


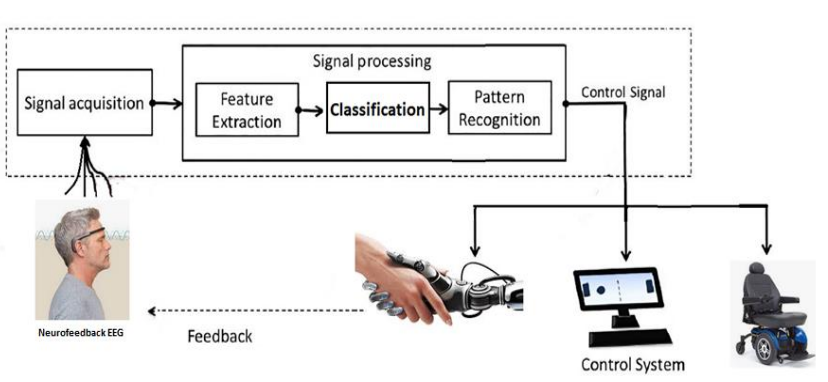

Fig. 3. BCI block diagram for EEGs processing.

\section{B. EEG signal Feature Classification}

According to recent research, a multi-domain feature set constituting time domain, frequency domain, and channel coherence features of EEG signal was extracted and quantified to map the attained neural responses into consequent emotional states[6]. Time domain feature (DWT) for EEG discrete wavelet transforms, frequency domain feature such as power spectral density (PSD) and acceleration spectral density (ASD), and spatial domain feature efficiently combined EEG characteristics from more than one electrode in linear correlation have been applied to EEG signal processing. Since the features extracted directly from the EEG signal always have strong fluctuations and contain some information unrelated to the emotion task, we should smooth the features by using the linear dynamical system (LDS) approach [7]. Therefore, the main task of the feature extraction stage was to derive the discrete wavelet features which can map the EEG data into corresponding emotional states.

\section{EEG signal Analysis and Pattern Recognition}

For classification of EEG wavelets into emotion categories or pattern recognition, it is necessary to extract features from wavelet and analyze nonlinear dynamical features as well. PC machine robots can use their fast massive parallel electronic computing or GPU with machine learning algorithms to analyze the data of extracted features after the EEG data acquisition and signal band filtering procedures in Fig. 3. In addition, it's necessary to reduce the feature dimensionality in order to improve or enhance the efficiency of the machine learning and prediction performance. There are three dimensionality reduction methods: principal component analysis (PCA), linear discriminant analysis (LDA) and correlation-based feature selector (CFS). Furthermore, to assess the association between EEG feature and emotional states, the classification into predefined emotional patterns was achieved by using support vector machine (SVM) classifiers and Fisher's discriminant analysis (FDA) as linear classifier or dimensionality reduction[8].

\section{RESEARCH METHODOLOGY AND EXPERIMENT DATA ANALYSIS}

According to the BCI system and EEG signal description before, the various spatial frequencies were generated by neuron activities. EEG studies mostly investigated classification of human emotion due to the poor spatial resolution of EEG wavelet recording. EEG signal is not exactly useful for pinpointing the exact sources of the activity and does not distinguish between human being emotions and trend actions. In this section, the research methodology focuses on EEG wavelet transform at minimum free energy (MFE) operated effortlessly in the visualization algorithm, and along with the assessment experiments.

\section{A. Research Methodology}

The research methodology for the EEG feature wavelet transform at minimum free energy (MFE) based on the thermodynamic equilibrium of human brain thermal reservoir at a constant brain temperature $\left(T_{0}=310^{\circ} \mathrm{K}\right.$ or $\left.37^{\circ} \mathrm{C}\right)$.

Since Boltzmann's entropy formula:

$S=K_{B} \log W$

where,

$K_{B}$ - Boltzmann's constant;

$W$ - the probability of macrostate occurrence for machine learning algorithm.

According to the $3^{\text {rd }}$ law of thermodynamics, the thermodynamic equilibrium is derived in terms of exponential weighted Helmholtz Free Energy of the brain.

$H_{\text {brain }}=E_{\text {brain }}-T_{0} S_{\text {brain }}$

where,

$H_{\text {brain }}$-Helmholtz free energy of the brain;

$E_{\text {brain }}$ - energy of the brain environment;

$T_{0}$ - Kelvin temperature $\left(310^{\circ} \mathrm{K}\right)$;

$S$ - Boltzmann's entropy.

Since Kelvin temperature can never be absolute zero, incessant molecular collisions will mix toward maximum uniformity as the Ludwig Boltzmann heat death due to the irreversible increase of the entropy interpreted as a measure of statistical disorder of a thermal system $\left(\Delta S_{\text {entropy }}>0\right)$.

Typically, the brain thermodynamic supported by homeostasis at Helmholtz Minimum Free Energy (MFE) should keep at a constant temperature $\left(T_{0}=37^{\circ} \mathrm{C}\right)$ to make effortless decisions. Therefore, it is referred to as normal emotions as opposed to other emotional states in the sense of a non-contrived straight forward decision such as loneliness, happiness and sadness. The exhaustive energy of the MFE is expressed in the following equation.

$\Delta H_{\text {brain }}=\Delta E_{\text {brain }}-T_{0} \Delta S_{\text {brain }}$

where,

$\Delta H_{\text {brain }}$ - the energy of MFE;

$\Delta E_{\text {brain }}$ - the instance energy of the environment;

$\Delta S_{\text {brain }}$ - the differential emotion state entropy.

For a measure of statistical disorder of a thermal system, $\Delta H_{\text {brain }}$ is always less than zero due to the differential entropy $\Delta S_{\text {entropy }}>0$ according to the equation (3). In our preview research, the brain activities at MFE can be illustrated on spectrogram. Fig. 4 shows the spectrograms of the differential brain activities at MFE. 


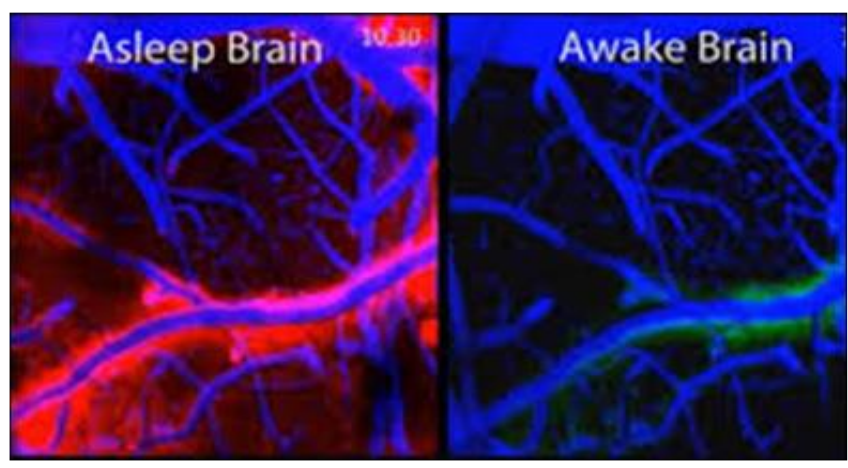

Fig. 4. The spectrograms of the asleep \& awake brain.

\section{B. Experiments for the Research}

In this paper, we will use EEG discrete wavelet transform at the minimum free energy which is opposite to operate effortlessly to measure the free energy of the emotion states. The experimental procedure is illustrated in Fig. 5.

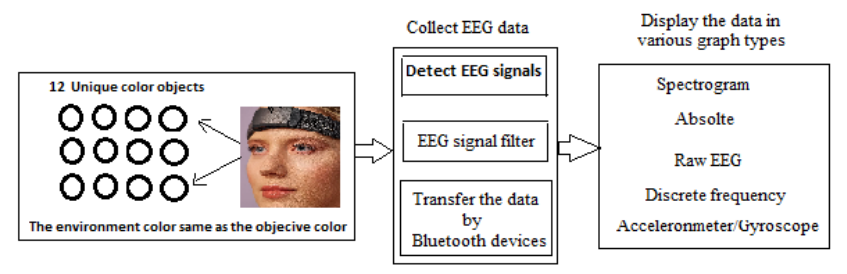

Fig. 5. EEG signal processing.

It is well known, different colors can affect human emotion when viewed, such as red for excitement, gray for loneliness, and clear for drowsiness. The EEG signals at MFE in the spectrogram and absolute signal in $\mathrm{dB}$ will be different from each other based on the color being observed. According to the EEG extracted data at MFE, we can identify the human emotion such as excitement, loneliness and drowsiness. We will examine the EEG spectrogram and absolute signals for the red, gray and clear color circumstances as the extracted data for excitement, loneliness and drowsiness. In this project, we will use 12 objects in red, gray and clear colors to influence a subject's emotion. A Muse headband will be used to receive the EEG signals, and Mind Monitor will showed the EEG Spectrogram and Absolute signals.

\section{Experiment Results and Analysis}

The main objective of this study is to estimate the different emotion states with BCI system communication and classify the human emotion features from EEG using discrete wavelet transform at MFE. Though, EEG signal handling is a tough task due to the external noise and the complexity of the signals, especially through non-invasive methodology. It is required to pre-process the EEG data for further analysis.

In Fig. 6, it shows the EEG signals of the clear color at MFE. Fig. 6(a) shows 12 samples in clear. Fig. 6(b) shows the absolute graphics of Delta, Theta, Alpha, Beta, and Gamma wavelets. When comparing these wavelet signals it can be seen that the delta decibel $(\mathrm{dB})$ and the theta decibel (dB) are higher than others. Fig. 6(c) shows MFE on energy spectrogram. The red color on spectrogram shows more energy consumption in the frequency range of $0 \mathrm{~Hz} \sim 8 \mathrm{~Hz}$. It means the brain is more motivated at the lower wavelet frequency.

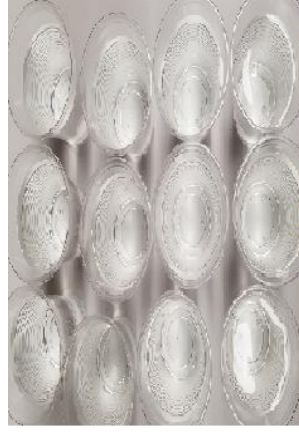

(a) Samples

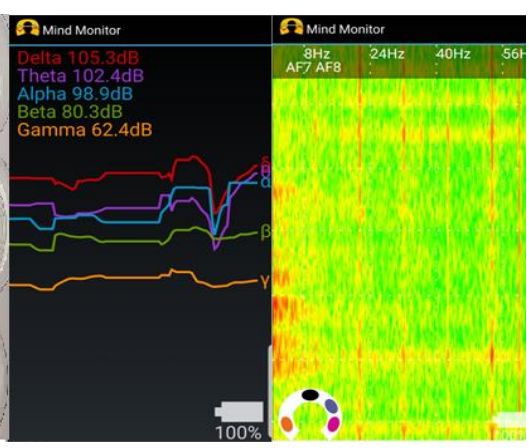

(b) Absolute

(c) Spectrogram

Fig. 6. The drowsiness emotion in clear.
In Fig. 7, it shows the EEG signals of the red color at MFE. Fig. 7(a) shows 12 objects in red. The dB of Beta and Gamma signal are higher than others in Fig. 7(b). Fig. 7(c) shows MFE on energy spectrogram. The red color on spectrogram graph shows more energy consumption in the frequency range of $24 \sim 40 \mathrm{~Hz}$. It means the brain is more motived and the frequency is higher. Since two sensors of the device didn't contact the surface of scalp well, there is more red color shown between $0 \mathrm{~Hz}$ and $8 \mathrm{~Hz}$. The sensor contact status is indicated at the left corner of the MFE spectrogram.



(a) Samples

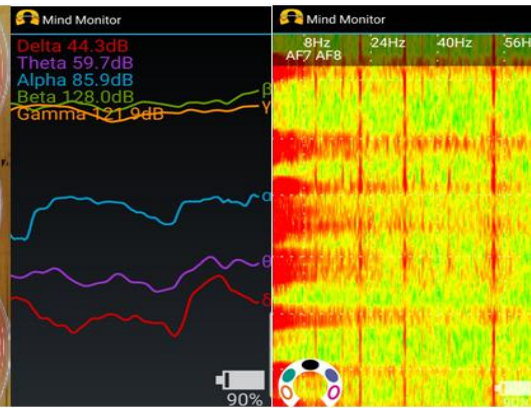

(b) Absolute (c) Spectrogram
Fig. 7. The excitement emotion in red color.

In Fig. 8, it shows the EEG signals of the gray color at MFE. Fig. 8(a) shows 12 samples in the gray color. Figure 8(b) shows the absolute graph of EEG signals. A comparison of the $\mathrm{dB}$ shows the $\mathrm{dB}$ of Alpha and Beta signal are higher than others. Fig. 8(c) shows the MFE on spectrogram graph. The spectrogram shows more energy consumption in the frequency range of $20 \mathrm{~Hz} 30 \mathrm{~Hz}$. The last bold red line shows the sensors didn't contact on the left of the forehead.

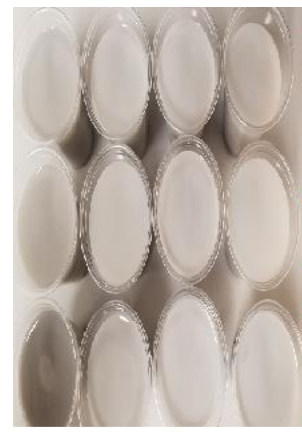

(a) Samples

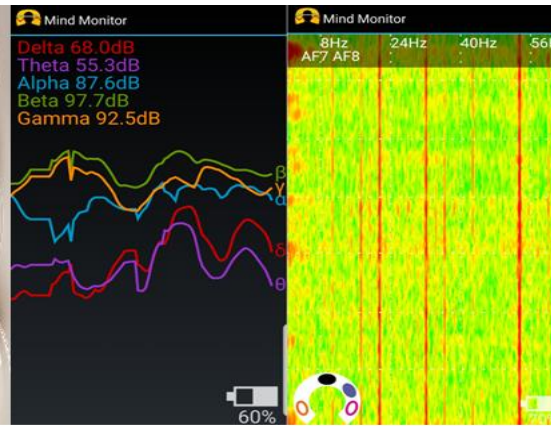

(b) Absolute

(c) Spectrogram
Fig. 8. The depression emotion in gray.

Hence, the different circumstances with a unique color hint the remarkable emotion states through the human being communication. 


\section{DISSCUSSION AND FUTURE WORK}

In a BCI system, EEG signals such as Delta wavelet signals are too hard to detect accurately due to the lower frequency. The neural cells of the brain never stop their activities even the human being is asleep. In this project, we used the discrete wavelet transform at minimum free energy operated effortlessly in order to evaluate the MFE of EEG signals in different emotional states. The MFE of the emotional states should be shown on spectrogram in red according to the scaled entropy. On the other hand, the external noises affect the wavelet signals easily due to the signal-noise-ratio (SNR) of the wavelet frequency. It has been shown in Fig. 7 (c). A lot of noise showed in the range of $0 \mathrm{~Hz} 3 \mathrm{~Hz}$ roughly due to two sensors contact issues.

The challenges of future work should strengthen the MFE of EEG wavelet signals. Recently, the spatial filters for the classification of event-related potentials have been applied to EEG signals [9]. After evaluating the decibels (dB) of EEG discrete wavelet signals, the human emotion features have been identified by machine learning (ML). Furthermore, the neuroscientists have used these methodologies for health clinics. The engineers will help the disabled people to control their wheel chairs or other devices.

\section{CONCLUSION}

The paper presented an approach which allows us to use EEG wavelet transform at a minimum free energy operated effortlessly for the classification of human emotion features in a BCI system. The results have been shown on the energy spectrogram graphics and EEG absolute signal graphics. According to the data on the graphics, we roughly verified the equations in the paper because of the external noise effect in the system. Furthermore, it should be noted that BCI will be more widely used in clinical practice and disability assistance.

\section{REFERENCES}

[1] A. Abdelaziz, "Brain computer interface (BCI)", International Journal of Computer Science and Mobile Computing, vol.8, no. 9, pp. 49-60, September 2019.

[2] M. A. Lebedev, M. A. L. Nicolelis, "Brain-machine interfaces: past, present and future," TRENDS in Neurosciences, vol. 29, no. 9, pp. 536546, September 1, 2006.

[3] Shiv Kumar Mudgal, Suresh K Sharma, Jitender Chaturvedi, Anil Sharma, "Brain computer interface advancement in neurosciences: Applications and issues," Interdisciplinary Neurosurgery, vol 20, pp. 1-8, June 2020.

[4] Eric W. Sellers, Emanuel Donchin, "A P300-based brain-computer interface: Initial tests by ALS pations," Clinical Neurophysiology, vol. 117, pp. 538-548, 2006.

[5] F. Lotte, M. Congedo, A. Lécuyer, F. Lamarche and B. Arnaldi, "A Review of Classification Algorithms for EEG-based Brain-Computer Interfaces," Journal of Neural Engineering, vol 4, no. 2, pp. 1-23, 2007 June.

[6] Rashima Mahajan, Dipali Bansal, "Depression diagnosis and management using EEG based affective brain mapping in real time," Int. J. Biomedical Engineering and Technology, Vol. 18, No. 2, pp. 115-138, 2015.

[7] Ulrich Hoffmann, Jean-Marc Vesin, Touradj Ebrahimi "Recent Advances in Brain-Computer Interfaces," IEEE International Workshop on Multimedia Signal Processing, IEEE Xplore, November 2007.

[8] XiaoWei Wang, Dan Nie, BaoLiang Lu, "Emotional state classification from EEG data using machine learning approach," Neurocomputing, Vol. 129, pp. 94-106, April 2014.
[9] Ulrich Hoffmann, Jean-Marc Vesin, Touradj Ebrahimi, "Spatial filter for the classification of event-related potentials," ESANN'2006 proceedings-European Symposium on Artificial Neural Networks, Belgium, 26-28 April 2006.

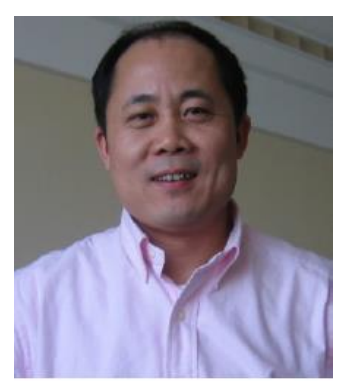

Hong Yu received the Bachelor degree in Electrical Engineering in 1989, M.S. degree in Electrical Engineering from the Catholic University of America in 2000 and his Ph.D. degree in Electrical Engineering from the CatholicUniversity of America in 2008. Currently, $\mathrm{He}$ is an assistant professor of Engineering Technology Department of Fitchburg State University. His teaching and interesting research areas include wireless network communication protocol, embedded system design, VLSI design and simulation, optical switching application, online Laboratory research and renewable energy development. He is IEEE senior member.

Sean-Paul Tomer is a student, He majors on electronic Engineering Technology at the department pf Engineering Technology in Fitchburg State University, USA. He is IEEE student member.

Marcus Perla is a student. He majors on Electronic Engineering Technology at the department of Engineering Technology in Fitchburg State University, USA. He is IEEE student member.

Yvette Yu graduated from University of Maryland. She major at finiance and information technology. She is Wall Street Fellow, Global Mindset Consulting Fellow and senior consultant in Protiviti golobel company. She is also fine artist. Some of her works exhibited in New York galleries. 